\title{
An Overview of In-memory Processing with Emerging Non-volatile Memory for Data-intensive Applications
}

\author{
Bing Li ${ }^{*}$ \\ bing.li.ece@duke.edu \\ ECE Dept., Duke University, US \\ Army Research Office, Research \\ Triangle Park, US
}

\author{
Bonan Yan \\ ECE Dept., Duke University \\ Durham, North Carolina, US \\ bonan.yan@duke.edu
}

\author{
Hai "Helen" Li \\ ECE Dept., Duke University \\ Durham, North Carolina, US \\ hai.li@duke.edu
}

\begin{abstract}
The conventional von Neumann architecture has been revealed as a major performance and energy bottleneck for rising data-intensive applications. The decade-old idea of leveraging in-memory processing to eliminate substantial data movements has returned and led extensive research activities. The effectiveness of in-memory processing heavily relies on the memory scalability, which cannot be satisfied by traditional memory technologies. Emerging non-volatile memories (eNVMs) that pose appealing qualities such as excellent scaling and low energy consumption, on the other hand, have been heavily investigated and explored for realizing in-memory processing architecture. In this paper, we summarize the recent research progress in eNVM-based in-memory processing from various aspects, including the adopted memory technologies, locations of the in-memory processing in system, supported arithmetics, as well as applied applications.
\end{abstract}

\section{CCS CONCEPTS}

- Hardware $\rightarrow$ Memory and dense storage; Spintronics and magnetic technologies; • Computer systems organization $\rightarrow$ Other architectures.

\section{KEYWORDS}

Data-intensive applications, emerging non-volatile memory, inmemory processing

\section{ACM Reference Format:}

Bing Li, Bonan Yan, and Hai "Helen" Li. 2019. An Overview of In-memory Processing with Emerging Non-volatile Memory for Data-intensive Applications. In Great Lakes Symposium on VLSI 2019 (GLSVLSI '19), May 9-11, 2019, Tysons Corner, VA, USA. ACM, New York, NY, USA, 6 pages. https://doi.org/10.1145/3299874.3319452

\section{INTRODUCTION}

In current era with data explosion, deep neural network (DNN) models are used to process various applications that explore a large

\footnotetext{
*This author is supported by NAS Associate Fellowship Award.

Permission to make digital or hard copies of all or part of this work for personal or classroom use is granted without fee provided that copies are not made or distributed for profit or commercial advantage and that copies bear this notice and the full citation on the first page. Copyrights for components of this work owned by others than ACM must be honored. Abstracting with credit is permitted. To copy otherwise, or republish, to post on servers or to redistribute to lists, requires prior specific permission and/or a fee. Request permissions from permissions@acm.org.

GLSVLSI '19, May 9-11, 2019, Tysons Corner, VA, USA

(c) 2019 Association for Computing Machinery.

ACM ISBN 978-1-4503-6252-8/19/05 . \$ \$15.00

https://doi.org/10.1145/3299874.3319452
}

amount of information in different data formats. Executing such data-intensive applications on conventional von Neumann systems causes massive data movements between processors and memory elements and induces significant performance and energy overheads. After decades since it was proposed first time [1, 2], the concept of in-memory processing returns and evokes many innovative solutions. Different from the conventional computing paradigm where data and computing are decoupled, in-memory processing architecture pulls data close to processing elements to reduce the amount of data movement and minimize the computation cost.

Benefiting from the recent advances in processing integration and memory technologies, many in-memory processing architectures have been developed. These attempts can be cataloged into three groups: (1) processing close to memory, aka near-data processing (NDP); (2) processing in traditional memory; and (3) processing in emerging non-volatile memory (eNVM).

In enabling NDP, three-dimension (3D) integration is a key technology. For example, 3D DRAM is constituted by vertically stacking a set of DRAM dies on top of a CMOS logic die over through-silicon vias (TSVs). There are a number of 3D DRAM-based NDP platforms that exploit the logic die to perform simple but common operations in data-intensive applications [16, 29-34]. TSVs substantially shorten the distance between the logic and memory dies, increasing the data bandwidth and improving overall performance.

Processing in memory directly performs computations in memory arrays so as to reduce data movement to a large extent. Prior works exploit traditional memory technologies such as DRAM and SRAM to complete the frequent operations appearing in dataintensive applications [35-39]. However, as the scaling of traditional memory technologies is approaching the physical limit, it is

Table 1: Emerging Non-volatile Memory Comparison

\begin{tabular}{cccccc}
\hline & SRAM & DRAM & STT-RAM & PCM & ReRAM \\
\hline $\begin{array}{c}\text { Cell Size } \\
\left(\mathrm{F}^{2}\right)\end{array}$ & $>100$ & $6-10$ & $6-50$ & $4-30$ & $\leq 2$ \\
\hline Multibit & 1 & 1 & 1 & $>2$ & $2-7$ \\
\hline $\begin{array}{c}\text { Endurance } \\
\text { Read Time } \\
(\text { ns) }\end{array}$ & $\sim 10^{16}$ & $>10^{16}$ & $>10^{15}$ & $10^{8}-10^{15}$ & $10^{8}-10^{12}$ \\
\hline $\begin{array}{c}\text { Write Time } \\
(\text { ns })\end{array}$ & $\sim 1$ & $\sim 10$ & $<10$ & $<10$ & $<10$ \\
\hline $\begin{array}{c}\text { Write Energy } \\
(\mathrm{J} / \text { bit })\end{array}$ & $\sim 10^{-15}$ & $\sim 10^{-14}$ & $\sim 10^{-13}$ & $\sim 10^{-11}$ & $\sim 10^{-13}$ \\
\hline & Source: [3-6]. Note: F represents the feature size. &
\end{tabular}


Table 2: An Overview of In-memory Processing Designs

\begin{tabular}{|c|c|c|c|c|c|c|}
\hline Works & & Types & Locations & Design Levels & Functions & Applications \\
\hline Guo et al. [7] & 2010 & \multirow{6}{*}{ STT-RAM } & Cache & Circuit; System & Logic; Arithmetic & Generic \\
\hline AC-DIMM [8] & 2013 & & Main Memory & Circuit; System & Associative & Generic \\
\hline Kang et al. [9] & 2017 & & - & Circuit & Logic & Bitmap \\
\hline STT-CiM [10] & 2018 & & Scratchpad & Circuit; System & Logic; Addition; Vector & Generic \\
\hline HielM [11] & 2018 & & - & Circuit; System & Logic & Encryption, Database \\
\hline Pan et al. [12] & 2018 & & Co-processor & Circuit; System & Logic & Binary CNN \\
\hline Cassinerio et al. [13] & 2013 & \multirow{7}{*}{ PCM } & - & Device & Logic & - \\
\hline Wright et al. $[14,15]$ & 2011, 2013 & & - & Device & Arithmetic & - \\
\hline Hosseini et al. [16] & 2015 & & - & Device & Arithmetic & - \\
\hline Pinatubo [17] & 2015 & & Main Memory & Circuit; System & Logic & Generic \\
\hline Burr et al. $[18,19]$ & 2015 & & - & Circuit & MVM & DNN \\
\hline Sebastian et al. [20] & 2017 & & - & Circuit & MVM & Unsupervised Learning \\
\hline Le et al. $[21,22]$ & 2017,2018 & & - & Circuit & MVM & Transfer Learning \\
\hline MAGIC [23] & 2014 & \multirow{6}{*}{ ReRAM } & Co-processor & Circuit & Logic; Arithmetic & Adder \\
\hline Bojnordi et al.[24] & 2016 & & Co-processor & System & MVM & Boltzmann machine \\
\hline ISAAC [25] & 2016 & & Co-processor & System & MVM & CNN \\
\hline PipeLayer [26] & 2017 & & Co-processor & System & MVM & CNN \\
\hline AtomLayer [27] & 2018 & & Co-processor & System & MVM & $\mathrm{CNN}$ \\
\hline GraphR [28] & 2018 & & Co-processor & System & MVM & Graph \\
\hline
\end{tabular}

Note: MVM - Matrix-Vector Multiplication; DNN - Deep Neural Network.

difficult to provide sufficient computing and storage capacity for data-intensive applications. Moreover, big cell size and high leakage power of traditional memory lead to large design area and energy consumption [40-42].

In recent years, eNVMs that demonstrate excellent scaling and near-zero leakage power are emerged as promising candidates for future trend. Table 1 compares traditional DRAM and SRAM with a few popular eNVM technologies, including spin-transfer torque RAM (STT-RAM), phase-change memory (PCM) and resistive RAM (ReRAM). Among these eNVM technologies, STT-RAM shows the fastest access speed and the lowest energy consumption while the cell area is relatively larger [41, 43]. Both PCMs [44, 45] and ReRAMs [46-48] can store multiple logic bits in a single memory cell, demonstrating superior density with technology scaling. In addition, they inherently support parallel data processing, which is uniquely beneficial to aforementioned data-intensive applications like DNNs. For the reason, extensive research efforts have been devoted to building in-memory processing using eNVM.

In this paper, we survey the recent progress in developing inmemory processing by leveraging the three mainstream eNVM technologies (STT-RAM, PCM and ReRAM). We present and discuss the difference and similarity of these works in terms of the supported functions, the location in architecture, the targeted applications, etc.

\section{DESIGN OVERVIEW}

Table 2 presents a summary of the latest eNVM-based in-memory processing designs reviewed in this paper. Each of them is classified according to the following five main categories.

- Type - The types of memory technologies adopted in these works: STT-RAM, PCM or ReRAM. Since the features of memories are different with each other, the selection of memory type determines the types of computation to some extent.
- Location - Where is the memory located in the computing architecture: cache, main memory or scratchpad? eNVMs can be used as storage and/or computing unit. Some works treat eNVM only as co-processor while some designate its location in memory hierarchy too.

- Design Level - The techniques in these works are carried out at different levels, such as device, circuit or system. Some works proposed the novel writing method to perform calculations in memory cells [13-16]. Some techniques are achieved through the modifications of the readout or write circuits associated with memory arrays. The system-level techniques would provide the interface and connection between the memory array and operation system so that the processing can be manipulated by applications.

- Functions - The function types in these works can be divided into the following groups: logic, arithmetic, associative, vector and matrix-vector multiplication (MVM). A type of operation can be realized by different eNVMs, but the implementation details could vary significantly.

- Applications - Most works provide advanced functions to support most data-intensive applications, which are grouped

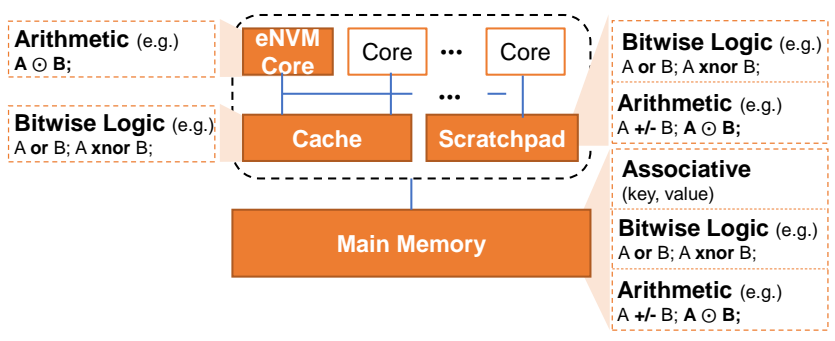

Figure 1: Processing locations and function types. 
into generic. Some works carry out only the core operation in a number of applications such as encryption, database, and CNN. A few works complete simple and basic operations that are not designated to any targeted applications.

Figure 1 depicts a high-level view of the classifications based on the location of the in-memory processing in system (eNVM core, cache, scratchpad memory, or main memory) and the type of supported functions (bitwise logic, arithmetic, or associative).

\section{ENVM-BASED IN-MEMORY PROCESSING \\ 3.1 Spin-Torque Transfer RAM (STT-RAM)}

STT-RAM consists of a magnetic tunnel junction (MTJ) device, which presents two resistance states depending on the relative magnetization orientation of the fixed and free ferromagnetic layers. Compared to other resistive memory devices, STT-RAM has faster write speed, lower write energy, and higher write endurance (refer Table 1). Due to the limited resistance difference between these distinct resistance states of MTJ, it is hard to implement multi-bit storage in STT-RAM cells. So most of STT-RAM-based in-memory processing designs focus on the bit-wise operations.

3.1.1 Associative and combinational logic. Early works exploit the high density of STT-RAM to complete the associative computing and combinational logic $[7,8]$. These works achieve reduced cost relative to traditional memory technologies. For instance, Guo et al. [7] employ STT-RAM to construct look-up table (LUT) and further realize the computing by cascading multiple LUTs. As such, the floating-point units are replaced by STT-RAM-based LUTs. The work successfully demonstrates the improved power and performance brought by STT-RAM technology, compared to multi-core CPU platform.

3.1.2 Bitwise logic operations. Recent works [9-11, 49] explore the use of STT-RAM in accomplishing bitwise logic operations. Based on the basic logic function realization by STT-RAM, advanced operations are implemented. Kang et al. [9], STT-CiM [10] and HieIM [11] are taken as examples and introduced here.

Kang et al. [9] propose a STT-RAM chip which can process bitwise logic and store information. The operands reside in different rows of the same array. By simultaneously activating multiple rows, the bitwise operations are enabled and results are obtained through the modified readout periphery circuits, i.e. sense amplifier (SA). The functionality of one logic operation can be controlled by modifying the bit in one control row. The chip can benefit some particular applications which involve intensive bitwise logic operations such as bitmap. This work focuses on circuit design and functional evaluation.

STT-CiM [10] extends the supported functions from bitwise logic to basic arithmetic and vector operations. At circuit level, the row decoders and SAs are enhanced to enable logic functions. Additional logic gates are integrated into the sense circuits to realize arithmetic operations. Two row decoders are used to active multiple rows where operands are located. The connected multiplex circuits are controlled by select signals to determine the desired operation type. When processing vector function, the vector outputs from STT-RAM arrays will be fed into reduction units and switched to the scalar value. At array level, authors analyze the impact of process variation on the computing results and deploy error correction scheme to enhance the reliability. At architectural and system levels, this work extends the instruction set to convey the operation command from applications to memory array. Through the extensions across multiple levels together, STT-CiM can be placed next to processor as on-chip scratchpad memory and applied for various data-intensive applications such as text processing, data compression, and digits recognition.

HieIM [11] implements bulk bitwise operations in STT-RAM array too. Different from the above two designs, HieIM is more flexible and allows the computing to operate between any cells within the same array. Moreover, a data encryption engine based on HieIM is demonstrated, which consumes $51.5 \%$ lower energy than the CMOS-based ASIC counterpart.

3.1.3 Neural networks. Thanks to the evolution of DNN models, convolution in binary convolutional neural network $(\mathrm{BCNN})$ can be replaced with bitwise operations such as XNOR and bit-count [50]. Pan et al. [12] build an accelerator based on multilevel STT-RAM (i.e. two-bit cell) for BCNN. STT-RAM arrays are programmable and can be switched between memory mode and bitwise operation mode. Thereby, multi-functional STT-RAM arrays are exploited to process convolutional layers. In this design, the two bits of one cell associates with inputs and weights, respectively so that the logic and add operations are carried out within one cell. This integrates the compuational STT-RAM array with an auxiliary processing unit (APU) which processes other computational layers in CNNs and implements the $\mathrm{BCNN}$ accelerator. Figure 2 illustrates the computing array and execution flows. Compared to other eNVM-based counterparts, the STT-RAM based accelerator achieves significant performance and energy improvement.

\subsection{Phase Change Memory (PCM)}

PCM can store more than one bit of data per cell, by diving the overall resistance range into a few levels. What's more, the cell conductance exhibits a linear increase along with the number of programming (more exactly, SET) pulses [51]. These key attributes of PCM are exploited to implement more complicate computations, such as the training of neural networks.

3.2.1 Logic and basic arithmetic operations. Phase change material manifests many physical attributes under various pulse amplitudes or duration, which have been exploited to realize computation. For example, Cassinerio et al. [13] leverage the resistance transition of phase change material and propose an initialize-compute-confirm scheme to implement Boolean logic operations within a single PCM cell. Wright et al. [14, 15] and Hosseini et al. [16] exploit the accumulative behavior of PCM material during programming and build an accumulator for arithmetic computations such as addition, subtraction, and parallel factoring. In these works, the partial and final results can be stored where computations are carried out. One single operation takes multiple cycles to complete as input operands sequentially enter. PCM cells are used to substitue for logic gates without revealing specific applications of interest.

Sebastian et al. [20] exploit the physical dynamics of PCM material and propose computational PCM to perform the temporal correlation detection between stochastic binary processes. One 


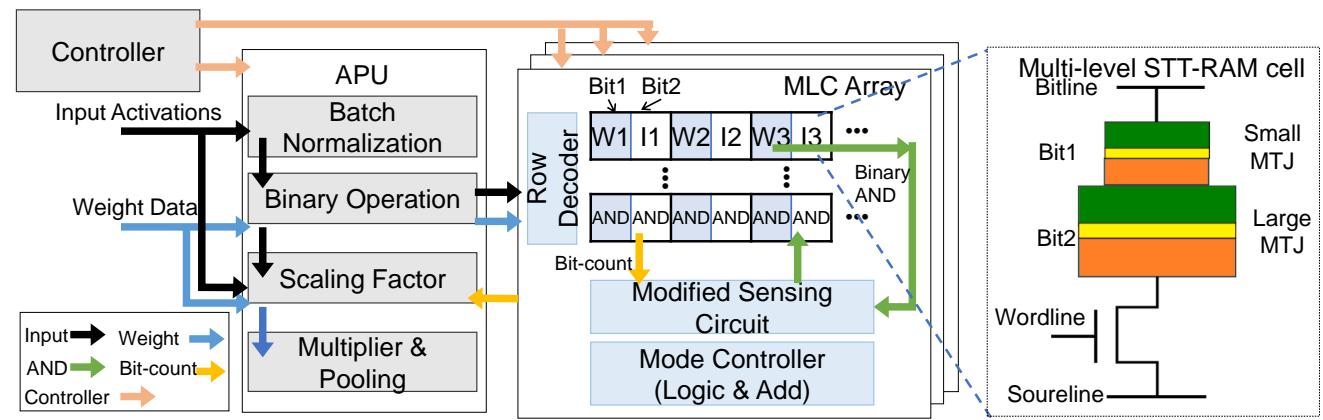

Figure 2: The execution flow, computing array, and multilevel STT-RAM cell for convolutional layers of Binary CNN in [12].

\begin{tabular}{|c|c|}
\hline \multicolumn{2}{|c|}{ Chip } \\
\hline \begin{tabular}{c} 
Controller \\
\hline $\begin{array}{c}\text { Output } \\
\text { Buffer }\end{array}$ \\
\hline $\begin{array}{c}\text { Inter-bank } \\
\text { operations }\end{array}$ \\
\hline
\end{tabular} Bank \\
\hline
\end{tabular}
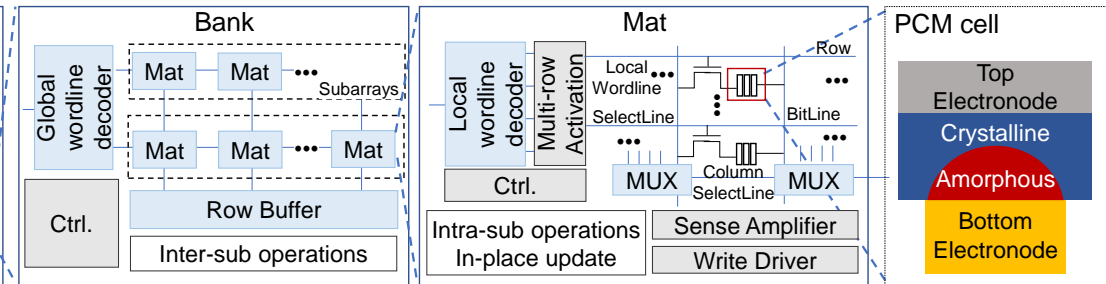

Figure 3: Pinatubo [17] and PCM cell architecture.

process is encoded into a SET pulse whose amplitude or duration is proportional to the instantaneous sum of all processes and enters the assigned PCM device. By comparing the conductance of each device, the correlated processes can be identified.

3.2.2 Matrix-vector multiplications \& machine learning. Arranged in the crossbar structure, PCM devices can process analog matrixvector multiplications, which have been intensively investigated [4, $18,19,21,22,52]$. An element in an matrix can be corresponded to the conductance of a PCM device. With an PCM crossbar representing a matrix, the vector is encoded into the amplitudes or duration of voltage pulses applied along rows. Then, the currents along columns will be proportional to the results. The positive and negative elements of the matrix could be stored in a pair of PCM devices. When applying input signals to columns, the currents along rows denote the results of the vector multiplying with the transposed matrix. A 3-layer perceptron using PCMs trained with backpropagation on the MNIST database of handwritten digits can achieve the comparable accuracy with the software model $[18,19]$. Moreover, leveraging PCM-based in-memory processing for other complicated tasks are demonstrated, such as compressed sensing recovery [22] and transfer learning [52].

3.2.3 System-level bitwise operations. Pinatubo [17] proposes a mechanism to perform bulk bitwise operations in PCM main memory. Read circuit and write driver is modified for Pinatubo processing logic functions. The operands are all stored in different rows in memory arrays. According to the locations that operands reside, Pinatubo has three computation modes: intra-subarray, intersubarray and inter-bank (Figure 3). The rows associated with operands will be activated simultaneously when computing. Sense amplifiers are enhanced with more reference circuits to obtain the logic outputs which will be sent to I/O bus or another memory row. To bridge operating system and logic operations inside PCM, Pinatubo develops the programming model and run-time supports to ensure that operands are allocated to different memory rows. The design achieves $1.12 \times$ overall speedup, $1.11 \times$ overall energy saving over the conventional CPU.

\subsection{Resistive RAM (ReRAM)}

The attractive features of high resistances and multi-level cell storage make ReRAM stand out from other emerging memory technologies to construct dense and low-power computing systems [53]. A variety of ReRAM based computing systems have been proposed to demonstrate superior performances in different applications central to memory access reduction.

3.3.1 Logic arithmetic operations. MAGIC [23] proposes mechanisms to perform bitwise operations with the aid of binary ReRAM. These schemes enable the integration of fundamental logic gates as well as complex arithmetic units, e.g. multi-bit full adders, within a ReRAM array.

3.3.2 Matrix-vector multiplications. ISAAC [25] is an neural network accelerator based on ReRAM dot-product engine. In ISAAC, ReRAM crossbar arrays both store weights in DNN and perform MVM with analog current and analog/digital converters (i.e. DAC $\&$ ADC). Figure 4 shows the top-down view of ISAAC chip. A group of tiles are connected through on-chip network. Every tile is composed of eDARM buffers, several in-situ multiply-accumulators (IMA), output registers and the shift-adders. Pooling and activation units in tiles dedicate to the pooling and activation operations in neural network. Each IMA consists of a number of ReRAM crossbars, ADCs, the input/output registers, and shift-adders. ISAAC exploits this integration of storage and computation for saving data (especially weights in filters) movement. The deeply pipelined flow of ISAAC focuses on the optimization in neural network inference.

A number of ReRAM-based CNN accelerators are proposed for boosting the system performance in training [26, 27, 54]. For example, PipeLayer [26] balances the parallelism and throughput in training and inference based on both parallelism granularity and weight duplication. By eliminating the potential stalls as in 

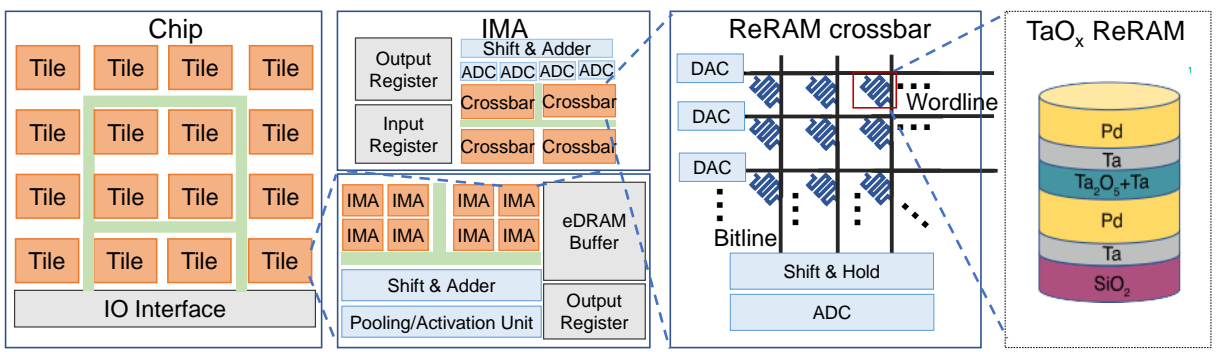

Figure 4: ISAAC [25], ReRAM crossbar, and ReRAM cell architecture.

ISAAC, PipeLayer yields an averagely $42.45 \times$ speedup and saves computation energy by $7.17 \times$ on average, compared to the massively parallel computing GPU platform. AtomLayer [27] attempts to provide a universal solution to enhance the efficiency during both training and inference. In this scheme, one network layer is executed at a time, i.e. atomic layer, to solve the issues brought by the highly pipelined operations, such as pipeline bubbles, long single-layer latency, and high cost of data buffers. AtomLayer revises the mapping scheme of weights to ReRAM arrays and data reuse and further reduces the on-chip data buffer access aside from the reduction of memory accesses. AtomLayer achieves $1.1 \times$ higher power efficiency than ISAAC in inference and $1.6 \times$ higher than PipeLayer in training, and its footprint shrinks $15 \times$ averagely with the reduction of on-chip buffers.

ReRAM array's parallel computing nature is capable of building accelerators for special computing models other than neural networks. GraphR [28] is a ReRAM-based graph processing accelerator to solve the poor locality and high-bandwidth requirement in graph processing. The ReRAM crossbar based graph engines offers low-cost hardware implementation to realize power-efficient graph processing acceleration.

Beyond convolutional computing engine, a number of works utilize ReRAM crossbars to support different computations and applications [24, 55-57]. For instance, Bojnordi et al. [24] implement the restricted Boltzmann machine with ReRAM arrays. The model of Boltzmann machine has been used to train deep neural networks with vast training samples. With the help of current summation circuit and reduction unit, large networks are reshaped to fit into ReRAM arrays, where in-situ computing operations are executed. Compared with conventional multi-core systems, ReRAM-based Boltzmann machine achieves $57 \times$ higher performance and $25 \times$ lower energy consumption without degrading the quality of solutions to optimization problems.

\section{CONCLUSION}

In this work, we gave an overview of recent works on eNVM-based in-memory processing that minimizes the cost of memory access and is expected to be meet the requirements of data-intensive applications. Emerging non-volatile memories (eNVMs) have advantages of low-power, high-density, superior scaling and inherent computing capability. Hence, numerous research works have been carried out to develop eNVM-based in-memory processing architectures. We summarize and discuss the types of eNVMs that have been adopted in in-memory processing designs, as well as a variety of implemented functions and supported applications. Because each type of eNVMs has distinct strengths and weaknesses, the selection of eNVM technology shall consider the specific requirements of applications. Following the progress of material science and device processing techniques, we anticipate continuous improvement in reliability, read/write speed, and energy efficiency of eNVM technologies. We believe the collaborative researches across various levels including device, circuit, system and applications, are essential to move eNVM-based in-memory processing towards commercial production.

\section{ACKNOWLEDGMENTS}

Bing Li acknowledges the National Academy of Sciences (NAS), USA for awarding the NRC research fellowship. Any opinions, findings and conclusions or recommendations expressed in this material are those of the authors and do not necessarily reflect the views of NAS or their contractors.

\section{REFERENCES}

[1] David Patterson, Thomas Anderson, Neal Cardwell, Richard Fromm, Kimberly Keeton, Christoforos Kozyrakis, Randi Thomas, and Katherine Yelick. A case for intelligent RAM. IEEE Micro, 17(2):34-44, 1997.

[2] Jeff Draper, Jacqueline Chame, Mary Hall, Craig Steele, Tim Barrett, Jeff LaCoss, John Granacki, Jaewook Shin, Chun Chen, Chang Woo Kang, et al. The architecture of the DIVAprocessing-in-memory chip. In Proceedings of the 16th international conference on Supercomputing (ICS), pages 14-25, 2002.

[3] Micron Technology Inc. Ddr4 sdram system-power calculator, June 2016.

[4] Geoffrey W Burr, Matthew J Brightsky, Abu Sebastian, Huai-Yu Cheng, Jau-Yi Wu, Sangbum Kim, Norma E Sosa, Nikolaos Papandreou, Hsiang-Lan Lung, Haralampos Pozidis, et al. Recent progress in phase-change memory technology. IEEE Journal on Emerging and Selected Topics in Circuits and Systems, 6(2):146-162, 2016.

[5] Sparsh Mittal, Jeffrey S Vetter, and Dong Li. A survey of architectural approaches for managing embedded dram and non-volatile on-chip caches. IEEE Transactions on Parallel and Distributed Systems, page 14, 2015.

[6] Shimeng Yu and Pai-Yu Chen. Emerging memory technologies: recent trends and prospects. IEEE Solid-State Circuits Magazine, 8(2):43-56, 2016.

[7] Xiaochen Guo, Engin Ipek, and Tolga Soyata. Resistive computation: avoiding the power wall with low-leakage, stt-mram based computing. ACM SIGARCH Computer Architecture News, 38(3):371-382, 2010.

[8] Qing Guo, Xiaochen Guo, Ravi Patel, Engin Ipek, and Eby G Friedman. Ac-dimm: associative computing with stt-mram. ACM SIGARCH Computer Architecture News, 41(3):189-200, 2013

[9] Wang Kang, Haotian Wang, Zhaohao Wang, Youguang Zhang, and Weisheng Zhao. In-memory processing paradigm for bitwise logic operations in stt-mram. IEEE Transactions on Magnetics, 53(11):1-4, 2017.

[10] Shubham Jain, Ashish Ranjan, Kaushik Roy, and Anand Raghunathan. Computing in memory with spin-transfer torque magnetic ram. IEEE Transactions on Very Large Scale Integration (VLSI) Systems, 26(3):470-483, 2018.

[11] Farhana Parveen, Zhezhi He, Shaahin Angizi, and Deliang Fan. Hielm: Highly flexible in-memory computing using stt mram. In Design Automation Conference (ASP-DAC), 2018 23rd Asia and South Pacific, pages 361-366. IEEE, 2018.

[12] Yu Pan, Peng Ouyang, Yinglin Zhao, Wang Kang, Shouyi Yin, Youguang Zhang, Weisheng Zhao, and Shaojun Wei. A multilevel cell stt-mram-based computing inmemory accelerator for binary convolutional neural network. IEEE Transactions on Magnetics, 54(99):1-5, 2018.

[13] M Cassinerio, N Ciocchini, and D Ielmini. Logic computation in phase change materials by threshold and memory switching. Advanced Materials, 25(41):59755980, 2013. 
[14] C David Wright, Peiman Hosseini, and Jorge A Vazquez Diosdado. Beyond vonneumann computing with nanoscale phase-change memory devices. Advanced Functional Materials, 23(18):2248-2254, 2013.

[15] C David Wright, Yanwei Liu, Krisztian I Kohary, Mustafa M Aziz, and Robert J Hicken. Arithmetic and biologically-inspired computing using phase-change materials. Advanced Materials, 23(30):3408-3413, 2011

[16] Peiman Hosseini, Abu Sebastian, Nikolaos Papandreou, C David Wright, and Harish Bhaskaran. Accumulation-based computing using phase-change memories with fet access devices. IEEE Electron Device Letters, 36(9):975-977, 2015.

[17] Shuangchen Li, Cong Xu, Qiaosha Zou, Jishen Zhao, Yu Lu, and Yuan Xie Pinatubo: A processing-in-memory architecture for bulk bitwise operations in emerging non-volatile memories. In Proceedings of the 53rd Annual Design Automation Conference, page 173. ACM, 2016.

[18] Geoffrey W Burr, Robert M Shelby, Severin Sidler, Carmelo Di Nolfo, Junwoo Jang, Irem Boybat, Rohit S Shenoy, Pritish Narayanan, Kumar Virwani, Emanuele U Giacometti, et al. Experimental demonstration and tolerancing of a large-scale neural network (165 000 synapses) using phase-change memory as the synaptic weight element. IEEE Transactions on Electron Devices, 62(11):3498-3507, 2015.

[19] GW Burr, P Narayanan, RM Shelby, Severin Sidler, Irem Boybat, Carmelo di Nolfo, and Yusuf Leblebici. Large-scale neural networks implemented with non-volatile memory as the synaptic weight element: Comparative performance analysis (accuracy, speed, and power). In Electron Devices Meeting (IEDM), 2015 IEEE International, pages 4-4. IEEE, 2015.

[20] Abu Sebastian, Tomas Tuma, Nikolaos Papandreou, Manuel Le Gallo, Lukas Kull, Thomas Parnell, and Evangelos Eleftheriou. Temporal correlation detection using computational phase-change memory. Nature Communications, 8(1):1115, 2017.

[21] M Le Gallo, A Sebastian, G Cherubini, H Giefers, and E Eleftheriou. Compressed sensing recovery using computational memory. In Electron Devices Meeting (IEDM), 2017 IEEE International, pages 28-3. IEEE, 2017.

[22] Manuel Le Gallo, Abu Sebastian, Roland Mathis, Matteo Manica, Heiner Giefers, Tomas Tuma, Costas Bekas, Alessandro Curioni, and Evangelos Eleftheriou. Mixed-precision in-memory computing. Nature Electronics, 1(4):246, 2018.

[23] Shahar Kvatinsky, Dmitry Belousov, Slavik Liman, Guy Satat, Nimrod Wald, Eby G Friedman, Avinoam Kolodny, and Uri C Weiser. Magic-memristor-aided logic. IEEE Transactions on Circuits and Systems II: Express Briefs, 61(11):895-899, 2014.

[24] Mahdi Nazm Bojnordi and Engin Ipek. Memristive boltzmann machine: A hardware accelerator for combinatorial optimization and deep learning. In High Performance Computer Architecture (HPCA), 2016 IEEE International Symposium on, pages 1-13. IEEE, 2016.

[25] Ali Shafiee, Anirban Nag, Naveen Muralimanohar, Rajeev Balasubramonian, John Paul Strachan, Miao Hu, R Stanley Williams, and Vivek Srikumar. Isaac A convolutional neural network accelerator with in-situ analog arithmetic in crossbars. ACM SIGARCH Computer Architecture News, 44(3):14-26, 2016.

[26] Linghao Song, Xuehai Qian, Hai Li, and Yiran Chen. Pipelayer: A pipelined reram based accelerator for deep learning. In High Performance Computer Architecture (HPCA), 2017 IEEE International Symposium on, pages 541-552. IEEE, 2017.

[27] Ximing Qiao, Xiong Cao, Huanrui Yang, Linghao Song, and Hai Li. Atomlayer: a universal reram-based cnn accelerator with atomic layer computation. In Proceedings of the 55th Annual Design Automation Conference, page 103. ACM 2018.

[28] Linghao Song, Youwei Zhuo, Xuehai Qian, Hai Li, and Yiran Chen. Graphr: Accelerating graph processing using reram. In High Performance Computer Architecture (HPCA), 2018 IEEE International Symposium on, pages 531-543. IEEE, 2018

[29] Seth H Pugsley, Jeffrey Jestes, Huihui Zhang, Rajeev Balasubramonian, Vijayalakshmi Srinivasan, Alper Buyuktosunoglu, Al Davis, and Feifei Li. Ndc: Analyzing the impact of 3d-stacked memory+ logic devices on mapreduce workloads. In 2014 IEEE International Symposium on Performance Analysis of Systems and Software (ISPASS), pages 190-200. IEEE, 2014.

[30] Amin Farmahini-Farahani, Jung Ho Ahn, Katherine Morrow, and Nam Sung Kim Nda: Near-dram acceleration architecture leveraging commodity dram devices and standard memory modules. In High Performance Computer Architecture (HPCA), 2015 IEEE 21st International Symposium on, pages 283-295. IEEE, 2015.

[31] Dongping Zhang, Nuwan Jayasena, Alexander Lyashevsky, Joseph L Greathouse Lifan Xu, and Michael Ignatowski. Top-pim: throughput-oriented programmable processing in memory. In Proceedings of the 23rd international symposium on High-performance parallel and distributed computing, pages 85-98. ACM, 2014.

[32] Mingyu Gao and Christos Kozyrakis. Hrl: Efficient and flexible reconfigurable logic for near-data processing. In High Performance Computer Architecture (HPCA), 2016 IEEE International Symposium on, pages 126-137. Ieee, 2016.

[33] Mingyu Gao, Jing Pu, Xuan Yang, Mark Horowitz, and Christos Kozyrakis. Tetris: Scalable and efficient neural network acceleration with $3 \mathrm{~d}$ memory. ACM SIGOPS Operating Systems Review, 51(2):751-764, 2017

[34] Duckhwan Kim, Jaeha Kung, Sek Chai, Sudhakar Yalamanchili, and Saibal Mukhopadhyay. Neurocube: A programmable digital neuromorphic architecture with high-density $3 \mathrm{~d}$ memory. In Computer Architecture (ISCA), 2016 ACM/IEEE 43rd Annual International Symposium on, pages 380-392. IEEE, 2016.
[35] Shuangchen Li, Dimin Niu, Krishna T Malladi, Hongzhong Zheng, Bob Brennan, and Yuan Xie. Drisa: A dram-based reconfigurable in-situ accelerator. In Proceedings of the 50th Annual IEEE/ACM International Symposium on Microarchitecture, pages 288-301. ACM, 2017.

[36] Quan Deng, Lei Jiang, Youtao Zhang, Minxuan Zhang, and Jun Yang. Dracc: a dram based accelerator for accurate cnn inference. In Proceedings of the 55th Annual Design Automation Conference, page 168. ACM, 2018

[37] Jintao Zhang, Zhuo Wang, and Naveen Verma. In-memory computation of a machine-learning classifier in a standard 6t sram array. 7. Solid-State Circuits, 52(4):915-924, 2017

[38] Amogh Agrawal, Akhilesh Jaiswal, Chankyu Lee, and Kaushik Roy. X-sram: Enabling in-memory boolean computations in cmos static random access memories. IEEE Transactions on Circuits and Systems I: Regular Papers, 65(99):1-14, 2018.

[39] Mingu Kang, Sujan K Gonugondla, Ameya Patil, and Naresh R Shanbhag. A multi-functional in-memory inference processor using a standard 6t sram array. IEEE Journal of Solid-State Circuits, 53(2):642-655, 2018.

[40] Minesh Patel, Jeremie S Kim, and Onur Mutlu. The reach profiler (reaper): Enabling the mitigation of dram retention failures via profiling at aggressive conditions. ACM SIGARCH Computer Architecture News, 45(2):255-268, 2017.

[41] Sang Phill Park, Sumeet Gupta, Niladri Mojumder, Anand Raghunathan, and Kaushik Roy. Future cache design using stt mrams for improved energy efficiency: devices, circuits and architecture. In Proceedings of the 49th Annual Design Automation Conference, pages 492-497. ACM, 2012.

[42] Sparsh Mittal. A survey of architectural techniques for improving cache power efficiency. Sustainable Computing: Informatics and Systems, 4(1):33-43, 2014

[43] Nour Sayed, Rajendra Bishnoi, Fabian Oboril, and Mehdi B Tahoori. A crosslayer adaptive approach for performance and power optimization in stt-mram. In 2018 Design, Automation \& Test in Europe Conference \& Exhibition (DATE), pages 791-796. IEEE, 2018.

[44] T Nirschl, JB Philipp, TD Happ, Geoffrey W Burr, B Rajendran, M-H Lee, A Schrott, $M$ Yang, M Breitwisch, C-F Chen, et al. Write strategies for 2 and 4-bit multilevel phase-change memory. In Electron Devices Meeting, 2007. IEDM 2007. IEEE International, pages 461-464. IEEE, 2007.

[45] Bing Li, Yu Hu, Ying Wang, Jing Ye, and Xiaowei Li. Power-utility-driven write management for mlc pcm. ACM fournal on Emerging Technologies in Computing Systems ( EETC), 13(3):50, 2017

[46] H-S Philip Wong, Heng-Yuan Lee, Shimeng Yu, Yu-Sheng Chen, Yi Wu, Pang-Shiu Chen, Byoungil Lee, Frederick T Chen, and Ming-Jinn Tsai. Metal-oxide rram. Proceedings of the IEEE, 100(6):1951-1970, 2012.

[47] Cong Xu, Dimin Niu, Naveen Muralimanohar, Rajeev Balasubramonian, Tao Zhang, Shimeng Yu, and Yuan Xie. Overcoming the challenges of crossbar resistive memory architectures. In 2015 IEEE 21st International Symposium on High Performance Computer Architecture (HPCA), pages 476-488. IEEE, 2015.

[48] Bing Li, Bonan Yan, Chenchen Liu, and Hai Helen Li. Build reliable and efficient neuromorphic design with memristor technology. In Proceedings of the 24th Asia and South Pacific Design Automation Conference, pages 224-229. ACM, 2019.

[49] Akhilesh Jaiswal, Amogh Agrawal, and Kaushik Roy. In-situ, in-memory statefu vector logic operations based on voltage controlled magnetic anisotropy. Scientific reports, 8(1):5738, 2018.

[50] Mohammad Rastegari, Vicente Ordonez, Joseph Redmon, and Ali Farhadi. Xnornet: Imagenet classification using binary convolutional neural networks. In European Conference on Computer Vision, pages 525-542. Springer, 2016.

[51] Abu Sebastian, Manuel Le Gallo, Geoffrey W. Burr, Sangbum Kim, Matthew BrightSky, and Evangelos Eleftheriou. Tutorial: Brain-inspired computing using phase-change memory devices. Fournal of Applied Physics, 124(11):111101, 2018.

[52] Stefano Ambrogio, Pritish Narayanan, Hsinyu Tsai, Robert M Shelby, Irem Boybat, Carmelo Nolfo, Severin Sidler, Massimo Giordano, Martina Bodini, Nathan CP Farinha, et al. Equivalent-accuracy accelerated neural-network training using analogue memory. Nature, 558(7708):60, 2018.

[53] Bonan Yan, Chenchen Liu, Xiaoxiao Liu, Yiran Chen, and Hai Li. Understanding the trade-offs of device, circuit and application in reram-based neuromorphic computing systems. In Electron Devices Meeting (IEDM), 2017 IEEE International, pages 11-4. IEEE, 2017.

[54] Ming Cheng, Lixue Xia, Zhenhua Zhu, Yi Cai, Yuan Xie, Yu Wang, and Huazhong Yang. Time: A training-in-memory architecture for memristor-based deep neura networks. In Proceedings of the 54th Annual Design Automation Conference 2017, page 26. ACM, 2017.

[55] Bing Li, Linghao Song, Fan Chen, Xuehai Qian, Yiran Chen, and Hai Helen Li. Reram-based accelerator for deep learning. In 2018 Design, Automation \& Test in Europe Conference \& Exhibition (DATE), pages 815-820. IEEE, 2018.

[56] Shihui Yin, Xiaoyu Sun, Shimeng Yu, Jae-sun Seo, and Chaitali Chakrabarti. A parallel rram synaptic array architecture for energy-efficient recurrent neural networks. In 2018 IEEE International Workshop on Signal Processing Systems (SiPS), pages 13-18. IEEE, 2018.

[57] Zichen Fan, Ziru Li, Bing Li, Yiran Chen, and Hai Helen Li. Red: A reram-based deconvolution accelerator. In 2019 Design, Automation \& Test in Europe Conference \& Exhibition (DATE), 2019. 